\title{
La importancia de la metodología de la investigación en la carrera de Medicina en la Universidad Nacional de Rosario
}

\author{
Castillo Salazar, Isis Zazil-Ha \\ Universidad Nacional de Rosario, Argentina \\ isis.zazilha.castillos@gmail.com
}

\author{
Belloni, Nadia Laura \\ Universidad Nacional de Rosario, Argentina \\ nadialbelloni511@gmail.com
}

\begin{abstract}
Resumen - Kerlinger y Lee (2002) menciona que la investigación es y debe ser tan ordenada que el investigador pueda tener plena confianza en sus resultados, por esta razón, se utiliza a la metodología como pilar de toda investigación pues no dar lugar al error, se encarga de clasificar y filtrar la información de manera tal, que se obtenga el conocimiento de la forma más acertada posible al contexto histórico, social y cultural del investigador (p. 13). Todo conocimiento debe ser cuestionado y puesto a prueba, hecho que nos remite al análisis que realiza esta disciplina para evaluar la calidad de la información recabada, y no puede ser desligada del proceso obtención - renovación de conocimiento, pues se ha pospuesto desde los inicios en un segundo plano para comprender mejor la función de la metodología.
\end{abstract}

Palabras clave - Metodología; Investigación; Carrera de medicina;

\begin{abstract}
Kerlinger and Lee (2002) mention that research is and must be so orderly that the researcher can have full confidence in its results, for this reason, methodology is used as a pillar of all research because it does not give room for error, it is responsible for classifying and filtering the information in such a way that knowledge is obtained in the most accurate way possible to the historical, social and cultural context of the researcher (p. 13). All knowledge must be questioned and tested, a fact that refers us to the analysis carried out by this discipline to evaluate the
\end{abstract}

Interconectando Saberes, 2021

ISSN: 2448-8704

(c) EY-NC-ND quality of the information collected and cannot be separated from the process of obtaining - renewing knowledge, since it has been postponed from the beginning in the background to better understand the function of methodology.

Keywords - Methodology; Research; Medical career;

"En las ciencias, el pensamiento es progresivo: sus etapas más recientes corrigen a las anteriores e incluyen a las verdades que persisten de estas etapas iniciales".

Albert Einstein, 1940

\section{INTRODUCCIÓN}

El proceso de adquisición de conocimiento se encuentra presente en todos los aspectos de la vida del ser humano, hecho por el cual, no debe desligarse del mismo, pues, al tener la premisa de que el humano es un ente bio-psico-social, gran parte del desarrollo social se encuentra en constante renovación, siendo así, pilar importante de las sociedades desde el punto de vista y análisis de cada individuo. El conocimiento es:

Fecha de Recepción: 5 de enero de 2021

Fecha de Aceptación: 29 de enero de 2021 Fecha de Publicación: 31 de enero de 2021 
conjunto de procesos sistemáticos, críticos y empíricos que se aplican al estudio de un fenómeno o problema con el resultado (o el objetivo) de ampliar su conocimiento. Esta concepción se aplica por igual a los enfoques cuantitativo, cualitativo y mixto. Los fenómenos pueden ser tan variados como el universo mismo: comportamientos, sentimientos y emociones, enfermedades, procesos psicológicos, organizaciones sociales (comunidades, empresas, etc.), valores y actitudes de los individuos. (HernándezSampieri \& Mendoza-Torres, 2018, p. 4)

Sin embargo, este proceso se ve aprendido mediante disciplinas como la metodología, las cuales van más allá de aprender a realizar una cita, ya que, nos lleva a cuestionar y reflexionar sobre todo material que se adquiera, dando la capacidad de discernir entre la información que se encuentra con un fundamento sólido, y aquella que no. "Cuando decimos que la investigación científica es sistemática y controlada queremos decir, de hecho, que la investigación es tan ordenada que los investigadores pueden tener una confianza crítica en los resultados”. (Kerlinger \& Lee, 2002, p. 14)

Para así, poder reconocer y aplicar herramientas apropiadas durante una búsqueda que le permita encontrar literatura actualizada, siendo un proceso mecanizado, en el cual, el estudiante ve como mero requisito la elaboración de dicho área instrumental, cumplir con los criterios establecidos y aprender a buscar información, así como utilizar las reglas del Manual de Publicaciones de la American Psychological Association (APA, 2020) dándole al alumno las bases para poder adquirir las competencias metodológicas esperables a ser desarrolladas en esta disciplina.

El estilo de la APA proporciona una base para la comunicación académica efectiva porque ayuda a los autores a presentar sus ideas de manera clara, concisa y organizada. La uniformidad y la consistencia permiten a los lectores: a) centrarse en las ideas que se presentan en vez de en el formato y b) explorar rápidamente los puntos clave, los hallazgos y las fuentes. Las directrices de estilo alientan a los autores a divulgar plenamente la información esencial y permiten a los lectores prescindir de distracciones menores, como las incoherencias u omisiones en la puntuación, las mayúsculas, las citas de referencia y la presentación de estadísticas.

Cuando el estilo funciona mejor, las ideas fluyen lógicamente, las fuentes se acreditan adecuadamente y los artículos se organizan de forma previsible y coherente. Las personas son descritas utilizando un lenguaje que afirma su valor y dignidad. Los autores planifican el cumplimiento ético e informan de los detalles críticos de su protocolo de investigación para permitir que los lectores evalúen los resultados y que otros investigadores reproduzcan potencialmente los estudios. Las tablas y figuras presentan los datos de manera atractiva $y$ coherente.

Ya sea que utilice el estilo de la APA para una sola clase o a lo largo de su carrera, le animamos a reconocer los beneficios de un enfoque concienzudo de la escritura. Aunque las pautas abarcan muchas áreas y requieren tiempo y práctica para aprender, esperamos que proporcionen un equilibrio entre la direccionalidad y la flexibilidad y que con el tiempo se conviertan en algo natural. (American Psychological Association, 2020, p. viii) 
La metodología requiere de la evaluación, pues facilita el discernimiento entre la información que se recaba y puede ser utilizada, junto con aquella que no se encuentra correcta o completa.

El conocimiento científico es un elemento esencial en la cultura y el desarrollo de las naciones, llegando a permitir un alto nivel de calidad de vida aún en países con escasos recursos naturales.

Si bien el conocimiento puede generarse desde diferentes ámbitos, la Universidad constituye uno de los espacios fundamentales para la producción, preservación y transmisión de dicho conocimiento.

La Medicina es un área en constante desarrollo y avance. Como toda disciplina científica, el desafío de sus progresos depende de la investigación científica, ya que, gracias a la investigación, se incorporan a la disciplina nuevos conocimientos.

En razón de ello, la formación de los futuros profesionales en el área de la salud debe incluir, además de una sólida formación teóricoconceptual y técnico- específica, herramientas adecuadas para la construcción del conocimiento científico. (Pezzotto \& Montenegro, 2020, p. 7)

\section{Planteamiento}

La Guía de aprendizaje Metodología de la Investigación Científica de esta área instrumental dentro de la Facultad de Ciencias Médicas en la Universidad Nacional de Rosario (UNR) se encuentra enfocada en un recorrido académico durante el cual, los estudiantes deberán analizar la producción del conocimiento científico, conocer el proceso de investigación y su marco teóricoconceptual.
El conocimiento de la medicina también tiene una historia conceptual a partir de la cual se han desarrollado diferentes formas de definir sus objetos y sus formas de explicarlos. Desde la época de Hipócrates hasta nuestros días se han construido, defendido y modificado múltiples teorías acerca de la salud y de la enfermedad dentro de diferentes paradigmas que han dado lugar a diferentes metodologías de investigación. Estas se han ido multiplicando en pos de discernir el objeto complejo de la salud y de la enfermedad. (Pezzotto \& Montenegro, 2020, p. 14)

En el plan de estudios vigente en la actualidad ( 2001), en la Carrera de Medicina de la Facultad de Ciencias Médicas de la UNR, el Área instrumental transversaliza los ciclos Promoción de la salud " destinado a comprender la importancia de la promoción de la salud y organizar el desarrollo de los contenidos y habilidades en torno al eje "conocer para cuidar" y Prevención de la Enfermedad " organizado en torno al eje "conocer para evitar", propone el desarrollo de contenidos y habilidades específicas para valorar el ejercicio de una práctica centrada en la prevención, que se anticipe a "procesos deteriorantes o contravalores", las injurias, en tanto ponen en situación de riesgo o vulnerabilidad la salud y los mecanismos de defensa." (Resolución C.S. No 158/2001, p. 16)

Las Áreas consideradas como una división funcional, operativa, para el cumplimiento de objetivos comunes para la adquisición de conocimientos, habilidades y destrezas, tienen el propósito de integrar las disciplinas; abarcan un campo integrado de aspectos convergentes que exigen un trabajo interdisciplinario ... La de Formación instrumental refiere a la organización de un conjunto de contenidos que tienden al cumplimiento de objetivos comunes para la 
adquisición de conocimientos, habilidades y destrezas para operar con distintos lenguajes, desarrollar la investigación científica y el pensamiento reflexivo. (Resolución C.S. No 158/2001, p. 18)

En esta área se incluye Metodología de la investigación por lo tanto es importante que los docentes encargados de acompañar al estudiante en el proceso de enseñanza - aprendizaje, sepan proporcionar de forma adecuada una perspectiva que le dé al estudiante las herramientas que lo llevarán al discernimiento del conocimiento que utilizará a lo largo de su formación académica y durante el tiempo que desempeñe su profesión.

\section{Cualquier investigación--o cuantitativa 0} cualitativa-requiere de rigor científico y ético.

La rigurosidad no tiene nada que ver con la rigidez, puesto que el rigor se relaciona con el respeto por los elementos básicos de la investigación, donde se debe tener en cuenta el rigor desde lo epistémico, lo metodológico y lo teórico. El investigador debe ser consciente de su forma de ver el mundo y sus limitaciones, cumplir con las condiciones que exige el método y de la rigurosidad teórica de la cual surgen las preguntas esenciales para enunciar. (Ramírez Atehortúa \& Zwerg-Villegas, 2012, p. 94)

Este proceso inicia con la habilidad de ser críticos al momento de elegir con qué material se va a basar un estudio, "el conocimiento implica una relación entre dos elementos esenciales: sujeto y objeto. Entendido el sujeto como la persona que busca, obtiene o posee el conocimiento; y el objeto como el hecho, fenómeno, tema o materia que el sujeto estudia" (Arias, 2006, p. 12). El escrutinio funciona adecuadamente cuando un tercero evalúa la información, involucrando a los estudiantes en el proceso y guiándolos como pares que en un momento dado de su profesión o formación académica deberán ser quienes otorguen dicha crítica constructiva a sus pares y se construya un nuevo conocimiento a partir del anterior.

\section{DISCUSIÓN}

La metodología de la investigación es el parteaguas en el conocimiento médico, esta es el mediador que coadyuva al proceso de generación del conocimiento.

Atento a satisfacer el objetivo de "generar un nuevo modelo científico biomédico y social que proyecte y fundamente un nuevo paradigma educativo en función del individuo y la sociedad." (resolución CS No158/2001), se propicia el abordaje interdisciplinario en el cual estas se convocan para un abordaje y desde la complejidad de los diversos desafíos que se presentan.

Si bien las ciencias y los procesos de investigación que guardan estrecha relación con la salud pública tienen una venerable historia, de notable desarrollo, también lo es que estos logros han ocurrido principalmente en el contexto del paradigma de las ciencias clásicas o la ciencia normal. Al penetrar en los fenómenos sociales, en los cuales la vida humana se constituye de hecho en un aspecto diferenciador fundamental, hace que el éxito del paradigma de investigación disminuya, pues si bien ha funcionado adecuadamente en 
ciertos campos como los biomédicos, demográficos, epidemiológicos y clínicos, entre otros, se hace limitado, dado que no permite adentrarse en el fondo de las interpretaciones culturales, ni en el análisis de los comportamientos, actitudes, creencias, valores, percepciones de la salud enfermedad y sus determinantes. (Jara Navarro, 2009, p. 34)

Realizando un recorrido histórico de cómo se fueron gestando los primeros abordajes metodológicos relacionados al conocimiento de las Ciencias Biomédicas, podríamos sintetizar que:

Los primeros esfuerzos por "investigar" y conocer la actividad vital del organismo y de los animales se remontan a los albores de la civilización. Los conocimientos de los antiguos se basaban fundamentalmente en conjeturas, de aquí que las conclusiones sobre la estructura y funciones del organismo fueran inexactas o erróneas. Sin embargo, el poder de observación y tenacidad de trabajo de esos hombres hicieron posible que poco a poco fuera desarrollándose un cuerpo de conocimientos propio de lo que posteriormente serían las diferentes disciplinas que hoy conforman la medicina moderna. (Carrizo Estévez, 2010, p. 3)

Conforme a las primeras investigaciones en el área de las ciencias de la salud que fueron el pilar de la amplia gama de disciplinas que hoy estructuran a la medicina moderna, se observa la labor de cada uno de aquellos profesionales que fueron pioneros en fundamentar la relevancia de la investigación, siendo así, los parteaguas del escrutinio de los estudios científicos.
Los profesionales sanitarios, investigadores 0 gestores sanitarios, así como los mismos usuarios del sistema sanitario necesitan, de forma creciente, información clínica relevante sobre la que deben fundamentar sus propias decisiones. Sin embargo, no suele ser fácil o viable, escrutar toda la evidencia científica que existe sobre el tema de interés. Las revisiones de la literatura científica juegan un papel central en la práctica clínica. Debido a las limitaciones de las revisiones narrativas, se genera la necesidad de disponer de revisiones sistemáticas que permitan integrar eficientemente toda la información válida y proporcionar una base objetiva y fiable para tomar decisiones de manera racional. Las revisiones sistemáticas que son síntesis y análisis de la información con un enfoque práctico se basan en la mejor evidencia científica disponible, formulan preguntas definidas con claridad y utilizan métodos sistemáticos y explícitos para identificar y seleccionar estudios, evaluar éstos de manera crítica, extraer los datos de interés y analizarlos. (Bonfill \& Schapira, 2010, p. 186)

En el área de la investigación, se debe tener en cuenta que todo conocimiento, después de ser evaluado, se adecúa a las circunstancias de cada sociedad, de esta manera se enfoca en desarrollar planes y estrategias que lleven a la práctica la información recabada, sin dejar de lado que la misma se encuentra sujeta a los cambios sociales, políticos, culturales y tecnológicos que, a medida que avanzan y se transforman, dan soluciones de manera objetiva a los problemas colectivos en materia de salud.

La investigación en salud es objeto de preocupación no solo en nuestro ámbito local, sino también a nivel mundial por lo que es necesario identificar problemas de salud no 
resueltos, planear estrategias sanitarias y acciones de prevención de las enfermedades que ocurren con mayor frecuencia. Las organizaciones de Salud deben adecuarse al contexto y buscar la solución a problemas de salud colectivos, en este sentido, una línea de acción ... es la de impulsar fuertemente el desarrollo de la investigación en salud con la integración de grupos de investigación, así como la formación de nuevos investigadores, lo que trae consigo una plataforma de conocimientos científicos para apoyar las decisiones que sustentan la práctica de la medicina y por ende la mejora en la calidad de los procesos de atención a la salud. (Toledo-Ocampo, 2013, p. 1)

Asumiendo a la Institución educativa como un espacio donde se conjugan saberes y conocimientos provenientes del vertiginoso devenir del desarrollo científico y tecnológico y de los cambios de paradigma emergentes, es muy importante propiciar la circulación de saberes socialmente significativos y reconocer la historicidad de las ciencias que favorecen el abordaje del objeto de estudio de la Carrera de Medicina, el proceso de salud enfermedad atención.

El enfoque médico-biológico ha tenido un peso preponderante dada su capacidad explicativa (4) en el nivel individual, y por los aportes científicos que fielmente ha construido para entender $y$ resolver problemas de salud en los individuos; sin embargo, el traslado mecánico de explicaciones e intervenciones hacia los niveles particular y general, es decir, hacia los grupos y las colectividades humanas, ha mostrado su insuficiencia para explicar y generar intervenciones evidenciando la necesidad de incorporar otras racionalidades y cuerpos conceptuales. En estas condiciones en el ámbito social, se perfila que la contribución de las ciencias sociales es aún escasa, los modelos conceptuales de que se dispone son incompletos y las explicaciones no logran permear el arraigado discurso natural biológico; por ello, el debate en torno a la dimensión social sigue siendo un espacio prolífico para avanzar en propuestas que, sin ignorar los aportes existentes, indaguen en los planos conceptual, metodológico y empírico para mejorar nuestra comprensión del problema y especialmente para generar propuestas de acción que permitan incidir en los problemas de salud de los grupos humanos. (Delgado-Bravo et al., 2014, p. 240)

De la tensión que surge en la dialogicidad entre certidumbre-incertidumbre, del reconocer lo azaroso, lo contingente, emerge el concepto de complejidad en el vocabulario científico, intentando transformar el concepto de ciencia. "Esta idea de complejidad se visualiza como el

punto articular para las investigaciones fundamentales, (en tanto) conjunto teórico / metodológico / epistemológico, a la vez coherente y abierto... Se trata de intentar un discurso multidimensional ... abierto a la incertidumbre y a la trascendencia; no ideal/idealista, sabiendo que la cosa no será nunca totalmente encerrada en el concepto, el mundo jamás aprisionado en el discurso. (Morin, 1997, p. 76).

Los avances en la medicina dependen de la investigación y esta permite el bienestar de los pacientes, así como el correcto tratamiento de las enfermedades y el control de estas.

Todo avance metodológico y cognitivo en las ciencias médicas es fruto de la investigación, puesto que constituye una parte fundamental en la adquisición y generación de nuevos 
conocimientos. El ejemplo anterior ilustra la importancia que ésta tiene en el campo clínico, así como el impacto que ejerce sobre la salud de la población mundial. El reporte de la Organización Mundial de la Salud (OMS) del 2013, manifiesta la urgencia de invertir y desarrollar investigación en salud como parte fundamental en el mejoramiento de la calidad de vida de los seres humanos. Para ello, los futuros descubrimientos en medicina deben sustentarse en estudios clínico-experimentales, que involucren a los diferentes niveles de atención médica. (López-Ortiz \& Mazón-Ramírez, 2016, p. 73)

\section{CONCLUSIONES}

Al analizar y dar cuenta del papel que tiene en las ciencias el uso de la metodología de la investigación como parte del proceso de adquisición de conocimientos, podemos sostener que el escrutinio de la información posibilita que esta misma pueda renovarse de forma constante, permitiendo la circulación de esta.

Consideramos necesario que los alumnos reconozcan a la investigación como un área prioritaria en su formación como futuros profesionales, proponiendo además brindar herramientas que favorezcan la reflexión crítica que demandará su futuro desarrollo profesional. Se espera que los estudiantes puedan plantearse interrogantes y reconocer la estrategia metodológica más adecuada para responderlos. (Pezzotto \& Montenero, 2020, p. 7)

El área instrumental donde pertenece metodología de la investigación dentro de la facultad de ciencias médicas se encuentra enfocada en donde, los estudiantes deberán analizar la producción del conocimiento científico, conocer el proceso de investigación y su marco teórico-conceptual para así, poder reconocer y aplicar herramientas apropiadas para la búsqueda que le permita encontrar fuentes actualizadas, siendo un proceso, en el cual, el estudiante ve como cumplir con los criterios establecidos y aprender a buscar información, así como utilizar las normas APA.

\section{REFERENCIAS}

Arias, F. G. (2006). El proyecto de investigación, Introducción a la metodología científica. Editorial Episteme.

Bonfill, X., \& Schapira, P. (2010). Importancia de la medicina basada en la evidencia para la práctica clínica. Colombia Médica, 41(2), 186-193 https://www.redalyc.org/pdf/283/2831681701 2.pdf

Delgado-Bravo, A. I., Naranjo-Toro, M., Castillo, R., Basante, Y., \& Rosero-Otero, M. (2014). Tendencias de investigación en salud. Análisis y reflexiones. Aquichan, 14(2), 237250

https://www.redalyc.org/articulo.oa?id=741/ $\underline{74131358010}$

González Pedraza Avilés, Alberto, \& Velasco Jiménez, María Teresa (2008). La importancia de la investigación en el primer nivel de atención a la salud. Revista de Especialidades Médico-Quirúrgicas, 13(4), 149-152.

https://www.redalyc.org/articulo.oa? $\mathrm{id}=473 / 4$ 7326420001

Hernández-Sampieri, R., \& Mendoza-Torres, P. (2018). Metodología de la investigación: las rutas cuantitativa, cualitativa y mixta. Mcgraw-Hill Interamericana Editores, S.A. de C. $\mathrm{V}$.

Kerlinger, F. N., \& Lee, H. B. (2002). Investigación del comportamiento cuarta edición. McgrawHill / Interamericana de México

López-Ortiz, G., \& Mazón-Ramírez, J. J. (2016). La importancia de la investigación clínica en Medicina Familiar. Atención Familiar, 23(3):73-74. https://www.elsevier.es/esrevista-atencion-familiar-223-pdfS1405887116301274 
Morin E. (1997). Introducción al pensamiento complejo. Gedisa. Barcelona.

Pezzotto, S. M., \& Montenegro, S. M. (2020). Guía de aprendizaje Metodología de la Investigación Científica. Universidad nacional de Rosario.

Ramírez-Atehortúa, F. B., Zwerg-Villegas, A. M. (2012). Metodología de la investigación: más que una receta. AD-minister, 20, 91-111. https://www.redalyc.org/articulo.oa?id=3223/ 322327350004

Resolución Consejo Superior (CS) de la Universidad Nacional de Rosario (UNR). (2001) No 158/2001.

Toledo Ocampo, E. A. (2013). La importancia de la investigación en salud. Salud en Tabasco, $19(1), 1$.

https://www.redalyc.org/articulo.oa?id $=487 / 4$ $\underline{8727474001}$ 